Honam Mathematical J. 36 (2014), No. 3, pp. 569-596

http://dx.doi.org/10.5831/HMJ.2014.36.3.569

\title{
A NEW FORM OF FUZZY GENERALIZED BI-IDEALS IN ORDERED SEMIGROUPS
}

\author{
Hidayat Ullah Khan*, Nor Haniza Sarmin, and Asghar Khan
}

\begin{abstract}
In several applied disciplines like control engineering, computer sciences, error-correcting codes and fuzzy automata theory, the use of fuzzified algebraic structures especially ordered semigroups and their fuzzy subsystems play a remarkable role. In this paper, we introduce the notion of $\left(\in, \in \vee \overline{\mathrm{q}}_{k}\right)$-fuzzy subsystems of ordered semigroups namely $\left(\in, \in \vee \overline{\mathrm{q}}_{k}\right)$-fuzzy generalized bi-ideals of ordered semigroups. The important milestone of the present paper is to link ordinary generalized bi-ideals and $\left(\in, \in \vee \bar{q}_{k}\right)$-fuzzy generalized bi-ideals. Moreover, different classes of ordered semigroups such as regular and left weakly regular ordered semigroups are characterized by the properties of this new notion. Finally, the upper part of a $\left(\in, \in \vee \overline{\mathrm{q}}_{k}\right)$-fuzzy generalized bi-ideal is defined and some characterizations are discussed.
\end{abstract}

\section{Introduction}

Fuzzy set theory [1] is a useful tool to describe situations in which the data are imprecise or vague and handle such situations by attributing a degree to which a certain object belongs to a set. Further, utilizing this fundamental concept of fuzzy set Rosenfeld introduced the notion of fuzzy subgroup [2]. Bhakat and Das [3] generalized Rosenfeld's fuzzy group theory and defined $(\in, \in \vee \mathrm{q})$-fuzzy subgroup by utilizing the combine notions of "belongingness" and "quasi-coincidence" of fuzzy point

Received June 16, 2014. Accepted August 26, 2014.

2010 Mathematics Subject Classification. 08A72, 20N25, 06F05, $20 \mathrm{M} 12$.

Key words and phrases. Ordered semigroups, regular, left (right) regular, completely regular and weakly regular ordered semigroups, generalized bi-ideals, fuzzy left (right) ideals, fuzzy generalized bi-ideals, $\left(\bar{\epsilon}, \bar{\in} \vee \overline{\mathrm{q}}_{k}\right)$-fuzzy generalized bi-ideals, $\left(\bar{\epsilon}, \bar{\epsilon} \vee \overline{\mathrm{q}}_{k}\right)$-fuzzy left (right) ideals, $\left(\in, \in \vee \overline{\mathrm{q}}_{k}\right)$-fuzzy bi-ideals.

${ }^{*}$ Corresponding author 
and fuzzy set [4]. In addition, Davvaz and Khan [5] discussed some characterization regular ordered semigroups in terms of $(\alpha, \beta)$-fuzzy generalized bi-ideals, where $\alpha, \beta \in\{\in, \mathrm{q}, \in \vee \mathrm{q}, \in \wedge \mathrm{q}\}$ and $\alpha \neq \in \wedge \mathrm{q}$. Moreover, in the semigroup theory the notions of generalized fuzzy (interior, bi-, left, right, quasi) ideals was studied respectively in ([6-9]). Kazanchi and Yamak [7] gave $\left(\bar{\epsilon}, \bar{\epsilon} \vee \overline{\mathrm{q}}_{k}\right)$-fuzzy bi-ideals of a semigroup and in [10] Shabir et. al studied $\left(\bar{\epsilon}, \bar{\epsilon} \vee \bar{q}_{k}\right)$-fuzzy ideals, generalized bi-ideals and quasi-ideals of a semigroup and characterized regular semigroups by the properties of these ideals. The reader is referred to [11-21] for further study regarding $(\alpha, \beta)$-fuzzy subsets and its generalization.

The aim of this paper to investigate more general form of fuzzy generalized bi-ideals. In this connection the notion of $\left(\bar{\epsilon}, \bar{\epsilon} \vee \overline{\mathrm{q}}_{k}\right)$-fuzzy generalized bi-ideals of ordered semigroup is introduced. In addition, Characterizations of regular, left weakly regular ordered semigroups by means of $\left(\bar{\epsilon}, \bar{\epsilon} \vee \overline{\mathrm{q}}_{k}\right)$-fuzzy generalized bi-ideals and $\left(\bar{\epsilon}, \bar{\epsilon} \vee \overline{\mathrm{q}}_{k}\right)$-fuzzy biideals are discussed. Further, the concepts of $\left(\bar{\epsilon}, \bar{\epsilon} \vee \overline{\mathrm{q}}_{k}\right)$-fuzzy left (right) ideals are also presented and some related properties are discussed.

\section{Basic Definitions and Preliminaries}

By an ordered semigroup (or po-semigroup) we mean a structure $(S, \cdot, \leq)$ in which the following conditions are satisfied:

$(O S 1)(S, \cdot)$ is a semigroup,

(OS2) $(S, \leq)$ is a poset,

(OS3) $a \leq b \longrightarrow a x \leq b x$ and $a \leq b \longrightarrow x a \leq x b$ for all $a, b, x \in S$.

For subsets $A, B$ of an ordered semigroup $S$, we denote by

$$
\begin{aligned}
A B & =\{a b \in S \mid a \in A, b \in B\}, \\
(A] & =\{t \in S \mid t \leq h \text { for some } h \in A\} .
\end{aligned}
$$

If $A=\{a\}$, then we write $(a]$ instead of $(\{a\}]$. For any $A, B \subseteq S$ we have $A \subseteq(A],(A](B] \subseteq(A B]$ and $((A])=(A]$.

Let $(S, \cdot, \leq)$ be an ordered semigroup. A non-empty subset $A$ of $S$ is called a subsemigroup of $S$ if $A^{2} \subseteq A$. A non-empty subset $A$ of $S$ is called a left (right) ideal of $S$ if

(i) $(\forall a \in S)(\forall b \in A)(a \leq b \longrightarrow a \in A)$,

(ii) $A S \subseteq A(S A \subseteq A)$.

A non-empty subset $A$ of an ordered semigroup $S$ is called a generalized bi-ideal [22] of $S$ if

(i) $(\forall a \in S)(\forall b \in A)(a \leq b \longrightarrow a \in A)$,

(ii) $A S A \subseteq A$. 
A non-empty subset $A$ of an ordered semigroup $S$ is called a bi-ideal [23] of $S$ if

(i) $(\forall a \in S)(\forall b \in A)(a \leq b \longrightarrow a \in A)$,

(ii) $A^{2} \subseteq A$,

(iii) $A S A \subseteq A$.

Note that, every bi-ideal of $S$ is a generalized bi-ideal of $S$, but the converse is not true, as shown in [22].

An ordered semigroup $S$ is regular [23] if for every $a \in S$, there exists $x \in S$ such that $a \leq a x a$, or equivalently, we have (i) $a \in(a S a]$ $\forall a \in S$ and (ii) $A \subseteq(A S A] \forall A \subseteq S$. An ordered semigroup $S$ is called left (right) regular [23] if for every $a \in S$ there exists $x \in S$ such that $a \leq x a^{2}\left(a \leq a^{2} x\right)$, or equivalently, (i) $a \in\left(S a^{2}\right]\left(a \in\left(a^{2} S\right]\right) \forall a \in S$ and (ii) $A \subseteq\left(S A^{2}\right]\left(A \subseteq\left(A^{2} S\right]\right) \forall A \subseteq S$. An ordered semigroup $S$ is called left (right) simple [23] if for every left (right) ideal $A$ of $S$ we have $A=S$ and $S$ is called simple [23] if it is both left and right simple. An ordered semigroup $S$ is left (right) regular [23] if for every $a \in S$, there exists $x \in S$, such that $a \leq x a^{2}\left(a \leq a^{2} x\right)$, or equivalently, (i) $a \in\left(S a^{2}\right]\left(a \in\left(a^{2} S\right]\right) \forall a \in S$ and (ii) $A \subseteq\left(S A^{2}\right]\left(A \subseteq\left(A^{2} S\right]\right) \forall A \subseteq S$. An ordered semigroup $S$ is called completely regular [23] if it is left regular, right regular and regular. An ordered semigroup $S$ is called left weakly regular [22] if for every $a \in S$, there exist $x, y \in S$ such that $a \leq x a y a$, or equivalently, (i) $a \in\left((S a)^{2}\right] \forall a \in S$ and (ii) $A \subseteq\left((S A)^{2}\right]$ $\forall A \subseteq S$. Right weakly regular ordered semigroups are defined similarly. An ordered semigroup $S$ is called weakly regular if it is both a left and right weakly regular.

Note that if $S$ is commutative, then the concepts of regular and weakly regular ordered semigroups coincide.

By $B(a)(L(a), R(a)$ and $I(a))$ we mean the generalized bi-(left, right and two-sided) ideal of $S$ generated by $a(a \in S)$ denoted by

$$
\begin{aligned}
B(a) & =(a \cup a S a], L(a)=(a \cup S a], R(a)=(a \cup a S], \\
I(a) & =(a \cup S a \cup a S \cup S a S] \text { (see [22, 23]). }
\end{aligned}
$$

Now, we give some fuzzy logic concepts.

A function $F: S \longrightarrow[0,1]$ (unit closed interval) is called a fuzzy subset of $S$.

The study of fuzzification of algebraic structures has started in the pioneering paper of Rosenfeld [3] in 1971. Rosenfeld introduced the notion of fuzzy groups and successfully extended many results from groups in the theory of fuzzy groups. Kuroki [24] studied fuzzy ideals, fuzzy bi-ideals and semiprime fuzzy ideals in semigroups (also see [25-27]). 
If $F$ and $G$ are fuzzy subsets of $S$ then $F \preceq G$ means $F(x) \leq G(x)$ for all $x \in S$ and the symbols $\wedge$ and $\vee$ will mean the following fuzzy subsets are defined as follow for all $x \in S$ :

$F \wedge G: S \longrightarrow[0,1] \mid x \longmapsto(F \wedge G)(x)=F(x) \wedge G(x)=\min \{F(x), G(x)\}$, $F \vee G: S \longrightarrow[0,1] \mid x \longmapsto(F \vee G)(x)=F(x) \vee G(x)=\max \{F(x), G(x)\}$.

A fuzzy subset $F$ of $S$ is called a fuzzy subsemigroup if

$$
F(x y) \geq \min \{F(x), G(y)\} \text { for all } x, y \in S .
$$

A fuzzy subset $F$ of $S$ is called a fuzzy generalized bi-ideal [22] of $S$ if:

(i) $x \leq y \longrightarrow F(x) \geq F(y)$,

(ii) $F(x y z) \geq \min \{F(x), F(z)\}$ for all $x, y, z \in S$.

A fuzzy subset $F$ of $S$ is called a fuzzy left (right)-ideal [23] of $S$ if:

(i) $x \leq y \longrightarrow F(x) \geq F(y)$,

(ii) $F(x y) \geq F(y)(F(x y) \geq F(x))$ for all $x, y \in S$.

A fuzzy subset of $S$ is called a fuzzy ideal if it is both a fuzzy left and right ideal of $S$.

A fuzzy subsemigroup $F$ is called a fuzzy bi-ideal [23] of $S$ if:

(i) $x \leq y \longrightarrow F(x) \geq F(y)$,

(ii) $F(x y z) \geq \min \{F(x), F(z)\}$ for all $x, y, z \in S$.

Note that every fuzzy bi-ideal is a generalized fuzzy bi-ideal of $S$. But the converse is not true, as given in [22].

Let $F$ be a fuzzy subset of an ordered semigroup $S$, then for all $t \in(0,1]$, the set $U(F ; t)=\{x \in S \mid F(x) \geq t\}$ is called a level set of $F$.

Theorem 2.1. ([28]) A fuzzy subset $F$ of an ordered semigroup $S$ is a fuzzy left (right)-ideal of $S$ if and only if $U(F ; t)(\neq \emptyset)$ where $t \in(0,1]$ is a left (right)-ideal of $S$.

Theorem 2.2. ([5]) A fuzzy subset $F$ of an ordered semigroup $S$ is a fuzzy generalized bi-ideal of $S$ if and only if $U(F ; t)(\neq \emptyset)$ where $t \in(0,1]$ is a generalized bi-ideal of $S$.

Theorem 2.3. ([28]) A non-empty subset $A$ of an ordered semigroup $S$ is a left (right)-ideal of $S$ if and only if

$$
\chi_{A}: S \longrightarrow[0,1] \mid x \longmapsto \chi_{A}(x)= \begin{cases}1, & \text { if } x \in A, \\ 0, & \text { if } x \notin A .\end{cases}
$$

is a fuzzy left (right)-ideal of $S$. 
Theorem 2.4. ([5]) A non-empty subset $A$ of an ordered semigroup $S$ is a generalized bi-ideal of $S$ if and only if

$$
\chi_{A}: S \longrightarrow[0,1] \mid x \longmapsto \chi_{A}(x)= \begin{cases}1, & \text { if } x \in A, \\ 0, & \text { if } x \notin A .\end{cases}
$$

is a fuzzy generalized bi-ideal of $S$.

If $a \in S$ and $A$ is a non-empty subset of $S$. Then,

$$
A_{a}=\{(y, z) \in S \times S \mid a \leq y z\} .
$$

For any two fuzzy subsets $F$ and $G$ of an ordered semigroup $S$, the product $F \circ G$ is defined by:

$$
F \circ G: S \longrightarrow[0,1] \mid a \longmapsto(F \circ G)(a)=\left\{\begin{array}{cc}
\bigvee_{(y, z) \in A_{a}}(F(y) \wedge G(z)), & \text { if } A_{a} \neq \emptyset, \\
0, & \text { if } A_{a}=\emptyset .
\end{array}\right.
$$

Let $F$ be a fuzzy subset of $S$, then the set of the form:

$$
F(y):= \begin{cases}t \in(0,1], & \text { if } y=x, \\ 0, & \text { otherwise }\end{cases}
$$

is called a fuzzy point with support $x$ and value $t$ and is denoted by $(x ; t)[2]$. A fuzzy point $(x ; t)$ is said to belong to (quasi-coincident with) a fuzzy set $F$, written as $(x ; t) \in F((x ; t) \mathrm{q} F)$ if $F(x) \geq t(F(x)+t>1)$ [2]. If $(x ; t) \in F$ or $(x ; t) \mathrm{q} F$, then we write $(x ; t) \in \vee \mathrm{q} F$. The symbol $\in \vee \mathrm{q}$ means $\in \vee \mathrm{q}$ does not hold.

Generalizing the concept of $(x ; t) \mathrm{q} F$, in semigroups, Khan et. al. [9] defined $(x ; t) \mathrm{q}_{k} F$, as $F(x)+t+k>1$, where $k \in[0,1)$.

Throughout in this paper $S$ will denote an ordered semigroup unless otherwise specified.

\section{3. $\left(\bar{\epsilon}, \bar{\in} \vee \overline{\mathbf{q}}_{k}\right)$-fuzzy generalized bi-ideals}

In this section, we define a more generalized form of $(\alpha, \beta)$-fuzzy generalized bi-ideals of an ordered semigroup $S$ and introduce $\left(\bar{\epsilon}, \bar{\epsilon} \vee \overline{\mathrm{q}}_{k}\right)$ fuzzy generalized bi-ideals of $S$, where $\alpha \in\{\bar{\epsilon}, \overline{\mathrm{q}}, \bar{\epsilon} \wedge \overline{\mathrm{q}}, \bar{\epsilon} \vee \overline{\mathrm{q}}\}$ and $k$ is an arbitrary element of $[0,1)$ unless otherwise specified.

Definition 3.1. A fuzzy subset $F$ of $S$ is called a $\left(\bar{\epsilon}, \bar{\in} \vee \overline{\mathrm{q}}_{k}\right)$-fuzzy subsemigroup of $S$ if for all $x, y \in S$ and $t \in(0,1]$ the following holds:

$$
(x y ; t) \bar{\epsilon} F \longrightarrow(x ; t) \bar{\epsilon} \vee \overline{\mathrm{q}}_{k} F \text { or }(y ; t) \bar{\epsilon} \vee \overline{\mathrm{q}}_{k} F .
$$


Definition 3.2. A fuzzy subset $F$ of $S$ is called a $\left(\bar{\epsilon}, \bar{\epsilon} \vee \overline{\mathrm{q}}_{k}\right)$-fuzzy generalized bi-ideal of $S$ if for all $x, y, z \in S$ and $t \in(0,1]$ the following conditions hold:

(1) $(\forall x \leq y),(x ; t) \bar{\in} F \longrightarrow(y ; t) \bar{\in} \vee \overline{\mathrm{q}}_{k} F$,

(2) $(x y z ; t) \bar{\epsilon} F \longrightarrow(x ; t) \bar{\epsilon} \vee \overline{\mathrm{q}}_{k} F$ or $(y ; t) \bar{\epsilon} \vee \overline{\mathrm{q}}_{k} F$.

Lemma 3.3. For any fuzzy subset $F$ of an ordered semigroup $S$ and for all $x, y \in S$ and $t \in(0,1]$ the following conditions are equivalent:

(1a) $(\forall x \leq y),(x ; t) \bar{\in} F \longrightarrow(y ; t) \bar{\in} \vee \bar{q}_{k} F$,

(1b) $(\forall x \leq y), \max \left\{F(x), \frac{1-k}{2}\right\} \geq F(y)$.

Proof. $(1 a) \Longrightarrow(1 b)$ : Suppose that there exist $x, y \in S$ with $x \leq y$ such that

$$
\max \left\{F(x), \frac{1-k}{2}\right\}<F(y)
$$

then

$$
\max \left\{F(x), \frac{1-k}{2}\right\}<t \leq F(y) \text { for some } t \in\left(\frac{1-k}{2}, 1\right] .
$$

Shows that $(x ; t) \bar{\epsilon} F$ but $(y ; t) \in \wedge \mathrm{q}_{k} F$, a contradiction. Therefore we accept that

$$
\max \left\{F(x), \frac{1-k}{2}\right\} \geq F(y) \text { for all } x, y \in S, \text { with } x \leq y .
$$

$(1 b) \Longrightarrow(1 a)$ : Let $(x ; t) \bar{\in} F$ for all $x, y \in S$ such that $x \leq y$ and $t \in(0,1]$, then $F(x)<t$. If

$$
\max \left\{F(x), \frac{1-k}{2}\right\}=F(x),
$$

then $F(y) \leq F(x)<t$ follows that $(y ; t) \bar{\in} F$. On the other hand if

$$
\max \left\{F(x), \frac{1-k}{2}\right\}=\frac{1-k}{2},
$$

then $F(y) \leq \frac{1-k}{2}$. Suppose that $(y ; t) \in F$, then $t \leq F(y) \leq \frac{1-k}{2}$ follows that $(y ; t) \overline{\mathrm{q}} F$ consequently $(y ; t) \bar{\in} \vee \overline{\mathrm{q}} F$.

Lemma 3.4. Let $F$ be a fuzzy subset of an ordered semigroup $S$. Then the following conditions are equivalent for all $x, y \in S$ and $t \in$ $(0,1]$ :

(2a) $(x y ; t) \bar{\epsilon} F \Longrightarrow(x ; t) \bar{\epsilon} \vee \bar{q}_{k}$ or $(y ; t) \bar{\epsilon} \vee \bar{q}_{k}$,

(2b) $\max \left\{F(x y), \frac{1-k}{2}\right\} \geq \min \{F(x), F(y)\}$. 
Proof. $(2 a) \Longrightarrow(2 b)$ : Let $\max \left\{F(x y), \frac{1-k}{2}\right\}<\min \{F(x), F(y)\}$ for some $x, y \in S$, then

$$
\max \left\{F(x y), \frac{1-k}{2}\right\}<t \leq \min \{F(x), F(y)\} \text { for some } t \in\left(\frac{1-k}{2}, 1\right] .
$$

shows that $(x y ; t) \bar{\epsilon} F$ but $(x ; t) \in \wedge q_{k} F$ and $(y ; t) \in \wedge q_{k} F$ a contradiction. Hence

$$
\max \left\{F(x y), \frac{1-k}{2}\right\} \geq \min \{F(x), F(y)\} \text { for all } x, y \in S .
$$

$(2 b) \Longrightarrow(2 a)$ : If $(x y ; t) \bar{\epsilon} F$ for all $x, y \in S$ such that $x \leq y$ and $t \in(0,1]$, then $F(x y)<t$. Consider

$$
\max \left\{F(x y), \frac{1-k}{2}\right\}=F(x y)
$$

then

$$
\min \{F(x), F(y)\} \leq \max \left\{F(x y), \frac{1-k}{2}\right\}=F(x y)<t,
$$

follows that $(x ; t) \bar{\in} F$ or $(y ; t) \bar{\epsilon} F$. But if

$$
\max \left\{F(x y), \frac{1-k}{2}\right\}=\frac{1-k}{2},
$$

then

$$
\min \{F(x), F(y)\} \leq \max \left\{F(x y), \frac{1-k}{2}\right\}=\frac{1-k}{2} .
$$

Suppose that $(x ; t) \in F$ or $(y ; t) \in F$. Then $t \leq F(x)<\frac{1-k}{2}$ or $t \leq$ $F(y)<\frac{1-k}{2}$ follows that $F(x)+t<\frac{1-k}{2}+\frac{1-k}{2}=1-k$ or $F(y)+t<$ $\frac{1-k}{2}+\frac{1-k}{2}=1-k$. Hence $(x ; t) \bar{q}_{k} F$ or $(y ; t) \bar{q}_{k} F$. Thus $(x ; t) \bar{\epsilon} \vee \bar{q}_{k} F$ or $(y ; t) \bar{\in} \vee \bar{q}_{k} F$.

Lemma 3.5. Let $F$ be a fuzzy subset of an ordered semigroup $S$. Then for all $x, y, z \in S$ and $t \in(0,1]$, the following conditions are equivalent:

(3a) $(x y z ; t) \bar{\epsilon} F \Longrightarrow(x ; t) \bar{\epsilon} \vee \bar{q}_{k} F$ or $(z ; t) \bar{\epsilon} \vee \bar{q}_{k} F$,

(3b) $\max \left\{F(x y z), \frac{1-k}{2}\right\} \geq \min \{F(x), F(z)\}$.

Proof. Follows from the proofs of Lemma 3.3 and 3.4.

From Lemma 3.3 and 3.5, we have the following theorem:

Theorem 3.6. A fuzzy subset $F$ of $S$ is a $\left(\bar{\epsilon}, \bar{\epsilon} \vee \bar{q}_{k}\right)$-fuzzy generalized bi-ideal of $S$ if and only if it satisfies the condition (1b) and (3b). 
Definition 3.7. A fuzzy subset $F$ which is both a $\left(\bar{\epsilon}, \bar{\epsilon} \vee \overline{\mathrm{q}}_{k}\right)$-fuzzy generalized bi-ideal and a $\left(\bar{\epsilon}, \bar{\epsilon} \vee \overline{\mathrm{q}}_{k}\right)$-fuzzy subsemigroup of $S$ is called a $\left(\bar{\epsilon}, \bar{\in} \vee \overline{\mathrm{q}}_{k}\right)$-fuzzy bi-ideal of $S$.

From Lemma 3.3, 3.4 and 3.5, we have the following theorem:

Theorem 3.8. A fuzzy subset $F$ of $S$ is a $\left(\bar{\epsilon}, \bar{\epsilon} \vee \bar{q}_{k}\right)$-fuzzy bi-ideal of $S$ if and only if it satisfies the condition (1b), (2b) and (3b).

Theorem 3.9. Let $F$ be a fuzzy subset of an ordered semigroup $S$. Then $F$ is $\left(\bar{\epsilon}, \bar{\in} \vee \bar{q}_{k}\right)$-fuzzy generalized bi-ideal of $S$ if and only if

$$
U(F ; t)=\{x \in S \mid F(x) \geq t\} \neq \emptyset
$$

is generalized bi-ideal of $S$ for all $t \in\left(\frac{1-k}{2}, 1\right]$.

Proof. Let $F$ be $(\bar{\epsilon}, \bar{\epsilon} \vee \bar{q})$-fuzzy generalized bi-ideal of $S$ and $t \in$ $\left(\frac{1-k}{2}, 1\right]$ be such that $U(F ; t) \neq \emptyset$. Then by Lemma $3.3(1 \mathrm{~b})$,

$$
F(b) \leq \max \left\{F(a), \frac{1-k}{2}\right\} \text { for } a \leq b \in U(F ; t),
$$

follows that $t \leq F(b) \leq \max \left\{F(a), \frac{1-k}{2}\right\}$ that is $F(a) \geq t$ (as $t \in$ $\left.\left(\frac{1-k}{2}, 1\right]\right)$ hence $a \in U(F ; t)$.

Next, we let $a, c \in U(F ; t)$, then by Lemma $3.5(3 \mathrm{~b})$,

$$
\max \left\{F(a b c), \frac{1-k}{2}\right\} \geq \min \{F(a), F(c)\} \geq \min \{t, t\}=t .
$$

follows that $F(a b c) \geq t$ (as $\left.t \in\left(\frac{1-k}{2}, 1\right]\right)$, hence $a b c \in U(F ; t)$.

Conversely, let $U(F ; t)=\{x \in S \mid F(x) \geq t\} \neq \emptyset$ be a generalized bi-ideal of $S$ for all $t \in\left(\frac{1-k}{2}, 1\right]$. Let $a, b \in S$ with $a \leq b$ such that $F(b)>\max \left\{F(a), \frac{1-k}{2}\right\}$, then $F(b) \geq t_{0}>\max \left\{F(a), \frac{1-k}{2}\right\}$ for some $t_{0} \in\left(\frac{1-k}{2}, 1\right]$. This shows that $b \in U\left(F ; t_{0}\right)$ but $a \bar{\in} U\left(F ; t_{0}\right)$, a contradiction and hence $F(x) \leq \max \left\{F(y), \frac{1-k}{2}\right\}$ for all $x \leq y$.

Example 3.10. Consider the ordered semigroup $S=\{a, b, c, d\}$ with the following multiplication table "." and order relation " $\leq$ ":

$$
\begin{array}{|c|c|c|c|c|}
\hline \cdot & a & b & c & d \\
\hline a & a & a & a & a \\
\hline b & a & a & a & a \\
\hline c & a & a & b & a \\
\hline d & a & a & b & b \\
\hline
\end{array}
$$$$
\leq:=\{(a, a),(b, b),(c, c),(d, d),(a, b)\} .
$$ 
Then $\{a\},\{a, b\},\{a, c\},\{a, d\},\{a, b, c\},\{a, c, d\}$ and $\{a, b, c, d\}$ are generalized bi-ideals of $S$. However, $\{a, c\},\{a, d\}$ and $\{a, c, d\}$ are not biideals of $S$. Define a fuzzy subset $F$ of $S$ as follows:

$$
F: S \longrightarrow[0,1] \mid x \longmapsto F(x)= \begin{cases}0.50, & \text { if } x=a, \\ 0.10, & \text { if } x=b, \\ 0.30, & \text { if } x=c, \\ 0.40, & \text { if } x=d .\end{cases}
$$

Then

$$
U(F ; t)= \begin{cases}S, & \text { if } 0.00<t \leq 0.10, \\ \{a, c, d\}, & \text { if } 0.10<t \leq 0.30, \\ \{a, d\}, & \text { if } 0.30<t \leq 0.40, \\ \{a\}, & \text { if } 0.40<t \leq 0.50, \\ \emptyset, & \text { if } 0.50<t \leq 1.00\end{cases}
$$

Thus, by Theorem $3.9, F$ is a $\left(\bar{\epsilon}, \bar{\epsilon} \vee \overline{\mathrm{q}}_{k}\right)$-fuzzy generalized bi-ideal of $S$ for all $t \in\left(\frac{1-k}{2}, 1\right]$ with $k=0.3$.

Note that, every $\left(\bar{\epsilon}, \bar{\epsilon} \vee \overline{\mathrm{q}}_{k}\right)$-fuzzy bi-ideal of $S$ is a $\left(\bar{\epsilon}, \bar{\epsilon} \vee \overline{\mathrm{q}}_{k}\right)$-fuzzy generalized bi-ideal of $S$. However, the converse is not true, in general, as shown in the following example.

Example 3.11. Consider the ordered semigroup as shown in Example 3.10. Then $F$ is a $\left(\bar{\epsilon}, \bar{\epsilon} \vee \overline{\mathrm{q}}_{k}\right)$-fuzzy generalized bi-ideal of $S$ but $F$ is not a $\left(\bar{\epsilon}, \bar{\epsilon} \vee \overline{\mathrm{q}}_{k}\right)$-fuzzy bi-ideal of $S$ because by condition (2b) of Lemma 3.4 , we have

$$
\max \left\{F(x y), \frac{1-k}{2}\right\} \geq \min \{F(x), F(y)\} .
$$

If $x=y=d$ and $k=0.3$ then

$$
\max \left\{F(d d)=0.1, \frac{1-k}{2}=0.35\right\} \nsupseteq F(d)=0.4 .
$$

Proposition 3.12. If $F$ is $(\bar{\epsilon}, \bar{\epsilon} \vee \bar{q})$-fuzzy generalized bi-ideal of $S$ and

$$
F_{\frac{1-k}{2}}=\left\{a \in S \mid F(a)>\frac{1-k}{2}\right\},
$$

then $F_{\frac{1-k}{2}}$ is a generalized bi-ideal of $S$. 
Proof. Let $a, b \in S$ such that $a \leq b \in F_{\frac{1-k}{2}}$. Then by Lemma 3.3 (1b),

$$
\max \left\{F(a), \frac{1-k}{2}\right\} \geq F(b)>\frac{1-k}{2} .
$$

This implies $F(a)>\frac{1-k}{2}$ (since $\left.\frac{1-k}{2} \ngtr \frac{1-k}{2}\right)$ i.e. $a \in F_{\frac{1-k}{2}}$.

Next, we let $a, b, c \in S$ such that $a, c \in F_{\frac{1-k}{2}}$. Then by Lemma 3.5 $(3 \mathrm{~b})$,

$$
\begin{aligned}
\max \left\{F(a b c), \frac{1-k}{2}\right\} & \geq \min \{F(a), F(c)\} \\
& >\frac{1-k}{2} .
\end{aligned}
$$

From this we see that $F(a b c)>\frac{1-k}{2}$ (since $\frac{1-k}{2} \ngtr \frac{1-k}{2}$ ) and we write $a b c \in F_{\frac{1-k}{2}}$. Hence $F_{\frac{1-k}{2}}$ is a generalized bi-ideal of $S$.

Consider a fuzzy subset $F$ of $S$ and $t \in(0,1]$. We define two sets as in the following:

$$
Q^{k}(F ; t)=\left\{x \in S \mid[x ; t] q_{k} F\right\} \text { and }[F]_{t}^{k}=\left\{x \in S \mid[x ; t] \in \vee q_{k} F\right\} .
$$

Theorem 3.13. If $F$ is $\left(\bar{\epsilon}, \bar{\in} \vee \bar{q}_{k}\right)$-fuzzy generalized bi-ideal of $S$ and

$$
Q^{k}(F ; t)=\left\{x \in S \mid[x ; t] q_{k} F\right\} \neq \emptyset,
$$

then $Q^{k}(F ; t)$ is generalized bi-ideal of $S$ for all $t \in\left(\frac{1-k}{2}, 1\right]$.

Proof. Let $F$ be $(\bar{\epsilon}, \bar{\in} \vee \bar{q})$-fuzzy generalized bi-ideal of $S$. Let $a, b \in S$ such that $a \leq b \in Q^{k}(F ; t)$ and $t \in\left(\frac{1-k}{2}, 1\right]$, then by Lemma 3.3 (1b)

$\max \left\{F(a), \frac{1-k}{2}\right\} \geq F(b)>1-k-t>(1-k)-\left(\frac{1-k}{2}\right)=\frac{1-k}{2}$.

This shows $F(a)>1-k-t$ and we write $a \in Q^{k}(F ; t)$.

Next, we let $a, b, c \in S$ such that $a, c \in Q^{k}(F ; t)$, then by Lemma 3.5 $(3 b)$,

$$
\begin{aligned}
\max \left\{F(a b c), \frac{1-k}{2}\right\} & \geq \min \{F(a), F(c)\} \\
& >\min \{1-k-t, 1-k-t\} \\
& =1-k-t .
\end{aligned}
$$

This shows that $F(a b c)>1-k-t\left(\right.$ as $\left.1-k-t>\frac{1-k}{2}\right)$ and we write $a b c \in Q^{k}(F ; t)$. Hence $Q^{k}(F ; t)$ is generalized bi-ideal of $S$. 
From Theorem 3.9 and Theorem 3.13 we can prove the following Theorem.

Theorem 3.14. Let $F$ be a fuzzy subset of $S$. Then $F$ is $\left(\bar{\epsilon}, \bar{\epsilon} \vee \bar{q}_{k}\right)$ fuzzy generalized bi-ideal of $S$ if and only if $[F]_{t}^{k} \neq \emptyset \Rightarrow[F]_{t}^{k}$ is a generalized bi-ideal of $S$ for all $t \in\left(\frac{1-k}{2}, 1\right]$.

Proposition 3.15. Every $\left(\bar{\epsilon}, \bar{\epsilon} \vee \bar{q}_{k}\right)$-fuzzy generalized bi-ideal of a regular ordered semigroup $S$ is a $\left(\bar{\epsilon}, \bar{\epsilon} \vee \bar{q}_{k}\right)$-fuzzy bi-ideal of $S$.

Proof. Let $a, b \in S$ and $F$ be a $\left(\bar{\epsilon}, \bar{\epsilon} \vee \overline{\mathrm{q}}_{k}\right)$-fuzzy generalized bi-ideal of $S$. Since $S$ is regular, there exists $x \in S$ such that $b \leq b x b$. Then

$\max \left\{F(a b), \frac{1-k}{2}\right\}=F(a b) \geq F(a(b x b))=F(a(b x) b) \geq\{F(a) \wedge F(b)\}$.

This means that $F$ is a $\left(\bar{\epsilon}, \bar{\epsilon} \vee \overline{\mathrm{q}}_{k}\right)$-fuzzy subsemigroup of $S$. Thus $F$ is a $\left(\bar{\epsilon}, \bar{\epsilon} \vee \overline{\mathrm{q}}_{k}\right)$-fuzzy bi-ideal of $S$.

Proposition 3.16. Every $\left(\bar{\epsilon}, \bar{\epsilon} \vee \bar{q}_{k}\right)$-fuzzy generalized bi-ideal of a left weakly regular ordered semigroup $S$ is a $\left(\bar{\epsilon}, \bar{\epsilon} \vee \bar{q}_{k}\right)$-fuzzy bi-ideal of $S$.

Proof. Let $a, b \in S$ and $F$ be a $\left(\bar{\epsilon}, \bar{\in} \vee \overline{\mathrm{q}}_{k}\right)$-fuzzy generalized bi-ideal of $S$. Since $S$ is left weakly regular, there exist $x, y \in S$ such that $b \leq x b y b$. Then

$$
\max \left\{F(a b), \frac{1-k}{2}\right\}=F(a b) \geq F(a(x b y b))=F(a(x b y) b) \geq\{F(a) \wedge F(b)\} .
$$

This means that $F$ is a $\left(\bar{\epsilon}, \bar{\epsilon} \vee \overline{\mathrm{q}}_{k}\right)$-fuzzy subsemigroup of $S$. Thus $F$ is a $\left(\bar{\epsilon}, \bar{\epsilon} \vee \overline{\mathrm{q}}_{k}\right)$-fuzzy bi-ideal of $S$.

Remark 3.17. From Proposition 3.15 and 3.16, it follows that in regular and left weakly regular ordered semigroups, the concepts of $\left(\bar{\epsilon}, \bar{\epsilon} \vee \overline{\mathrm{q}}_{k}\right)$-fuzzy generalized bi-ideals and $\left(\bar{\epsilon}, \bar{\epsilon} \vee \overline{\mathrm{q}}_{k}\right)$-fuzzy bi-ideals coincide.

Lemma 3.18. A non empty subset $A$ of $S$ is generalized bi-ideal of $S$ if and only if the characteristic $\chi_{A}$ of $A$ is $\left(\bar{\epsilon}, \bar{\epsilon} \vee \bar{q}_{k}\right)$-fuzzy generalized bi-ideal of $S$.

Proof. The proof is obvious and is omitted.

Definition 3.19. A fuzzy subset $F$ of $S$ is called $\left(\bar{\epsilon}, \bar{\epsilon} \vee \bar{q}_{k}\right)$-fuzzy left (right) ideal of $S$ if

(i) $(\forall x, y \in S, t \in(0,1]$ and $x \leq y)(x ; t) \bar{\in} F \rightarrow(y ; t) \bar{\in} \vee \bar{q}_{k} F$,

(ii) $(\forall x, y \in S, t \in(0,1])(x y ; t) \bar{\epsilon} F \rightarrow(y ; t) \bar{\in} \vee \bar{q}_{k} F\left((x ; t) \bar{\in} \vee \bar{q}_{k} F\right)$. 
Theorem 3.20. A fuzzy subset $F$ of $S$ is $\left(\bar{\epsilon}, \bar{\epsilon} \vee \bar{q}_{k}\right)$-fuzzy left (right) ideal of $S$ if and only if the following conditions hold for all $x, y \in S$, $t \in(0,1]$ :

(iii) $\max \left\{F(x), \frac{1-k}{2}\right\} \geq F(y)$ for all $x \leq y$.

(iv) $\max \left\{F(x y), \frac{1-k}{2}\right\} \geq F(y)\left(\max \left\{F(x y), \frac{1-k}{2}\right\} \geq F(x)\right)$.

Proof. Let $F$ be $\left(\bar{\epsilon}, \bar{\epsilon} \vee \bar{q}_{k}\right)$-fuzzy left ideal of $S$ and

$$
\max \left\{F(a), \frac{1-k}{2}\right\}<F(b) \text { for some } a, b \in S \text { with } a \leq b .
$$

Then

$$
\max \left\{F(a), \frac{1-k}{2}\right\}<t \leq F(b) \text { for some } t \in\left(\frac{1-k}{2}, 1\right] .
$$

This show that $(a ; t) \bar{\epsilon} F$ but $(b ; t) \in F$, a contradiction and hence

$$
\max \left\{F(x), \frac{1-k}{2}\right\} \geq F(y) \text { for all } x \leq y .
$$

Next, we consider

$$
\max \left\{F(a b), \frac{1-k}{2}\right\}<F(b) \text { for some } a, b \in S .
$$

Then there exist some $t \in\left(\frac{1-k}{2}, 1\right]$ such that

$$
\max \left\{F(a b), \frac{1-k}{2}\right\}<t \leq F(b) .
$$

We see that $(a b ; t) \bar{\epsilon} F$ but $(b ; t) \in F$, a contradiction and thus

$$
\max \left\{F(x y), \frac{1-k}{2}\right\} \geq F(y) .
$$

Conversely, let (iii) and (iv) are satisfied for all $x, y \in S$. Let $x, y \in S$ with $x \leq y$ such that $(x ; t) \bar{\epsilon} F$. Then by (iii)

$$
\begin{aligned}
F(y) & \leq \max \left\{F(x), \frac{1-k}{2}\right\} \\
& = \begin{cases}F(x)<t, & \text { if } F(x) \geq \frac{1-k}{2} \\
\frac{1-k}{2}, & \text { if } F(x)<\frac{1-k}{2} .\end{cases}
\end{aligned}
$$

From here we observe that $(y ; t) \bar{\epsilon} F$ or $F(y)+t+k<1$ (if $\left.t \leq \frac{1-k}{2}\right)$ i.e. $(y ; t) \bar{q}_{k} F$. On the other hand, if $t>\frac{1-k}{2}$ then $(y ; t) \bar{\in} F$ and consequently $(y ; t) \bar{\in} \vee \bar{q}_{k} F$. 
Lastly we choose $x, y \in S$ such that $(x y ; t) \bar{\epsilon} F$, then by (iv),

$$
\begin{aligned}
F(y) & \leq \max \left\{F(x y), \frac{1-k}{2}\right\} \\
& = \begin{cases}F(x y)<t, & \text { if } F(x y) \geq \frac{1-k}{2}, \\
\frac{1-k}{2}, & \text { if } F(x y)<\frac{1-k}{2} .\end{cases}
\end{aligned}
$$

It follows that $(y ; t) \bar{\epsilon} F$ or $F(y)+t+k<1$ (if $t \leq \frac{1-k}{2}$ ) i.e. $(y ; t) \bar{q}_{k} F$. On the other hand, if $t>\frac{1-k}{2}$ then $(y ; t) \bar{\epsilon} F$. Hence $(y ; t) \bar{\epsilon} \vee \bar{q}_{k} F$. Consequently, $F$ is $\left(\bar{\epsilon}, \bar{\epsilon} \vee \bar{q}_{k}\right)$-fuzzy left ideal of $S$. Similarly one can show this for right ideal.

Theorem 3.21. A fuzzy subset $\lambda$ of $S$ is a $\left(\bar{\epsilon}, \bar{\epsilon} \vee \bar{q}_{k}\right)$-fuzzy left (right)-ideal of $S$ if and only if $U(\lambda ; t)(\neq \emptyset)$ is a left (right)-ideal of $S$ for all $t \in\left(0, \frac{1-k}{2}\right]$.

Proof. The proof follows from Theorem 3.9.

\section{Upper parts of $\left(\bar{\epsilon}, \bar{\epsilon} \vee \bar{q}_{k}\right)$-fuzzy generalized bi-ideals}

In this section, we define the upper/lower parts of an $\left(\bar{\epsilon}, \bar{\epsilon} \vee \bar{q}_{k}\right)$-fuzzy generalized bi-ideal and characterize regular and left weakly regular ordered semigroups in terms of $\left(\bar{\epsilon}, \bar{\epsilon} \vee \bar{q}_{k}\right)$-fuzzy generalized bi-ideals and $\left(\bar{\epsilon}, \bar{\epsilon} \vee \bar{q}_{k}\right)$-fuzzy left (right)-ideals.

Definition 4.1. ([10]) For any fuzzy subsets $F$ and $G$ of $S$, then for all $x \in S$ the fuzzy subsets $F^{+k},\left(F \wedge^{k} G\right)^{+},\left(F \vee^{k} G\right)^{+}$and $\left(F \circ^{k} G\right)^{+}$ of $S$ are defined as follows:

$$
\begin{aligned}
& F^{+k}: S \longrightarrow[0,1] \mid x \longmapsto F^{k}(x)=F(x) \vee \frac{1-k}{2}, \\
&\left(F \wedge^{k} G\right)^{+} \quad: \quad S \longrightarrow[0,1] \mid x \longmapsto\left(F \wedge^{k} G\right)(x)=(F \wedge G)(x) \vee \frac{1-k}{2}, \\
&\left(F \vee^{k} G\right)^{+} \quad: \quad S \longrightarrow[0,1] \mid x \longmapsto\left(F \vee^{k} G\right)(x)=(F \vee G)(x) \vee \frac{1-k}{2}, \\
&\left(F \circ^{k} G\right)^{+} \quad: \quad S \longrightarrow[0,1] \mid x \longmapsto\left(F \circ^{k} G\right)(x)=(F \circ G)(x) \vee \frac{1-k}{2} .
\end{aligned}
$$

Lemma 4.2. Let $F$ and $G$ be fuzzy subsets of $S$. Then the following hold:

(i) $\left(F \wedge^{k} G\right)^{+}=\left(F^{+k} \wedge G^{+k}\right)$,

(ii) $\left(F \vee^{k} G\right)^{+}=\left(F^{+k} \vee G^{+k}\right)$, 
(iii) $\left(F \circ^{k} G\right)^{+} \succeq\left(F^{+k} \circ G^{+k}\right)$, if $A_{x}=\emptyset$ and $\left(F \circ \circ^{k} G\right)^{+}=\left(F^{+k} \circ\right.$ $\left.G^{+k}\right)$, if $A_{x} \neq \emptyset$.

Proof. (i) and (ii) follows from [10, Proposition 13].

(iii) Let $a \in S$. If $A_{a}=\emptyset$, then $\left(F \circ^{k} G\right)^{+}(a)=(F \circ G)(a) \vee \frac{1-k}{2}=$ $0 \vee \frac{1-k}{2}=\frac{1-k}{2}$. On the other hand, $\left(F^{+k} \circ G^{+k}\right)(a)=0$ and hence $\left(F^{+k} \circ G^{+k}\right) \preceq\left(F \circ^{k} G\right)^{+}$. Let $A_{a} \neq \emptyset$, then

$$
\begin{aligned}
\left(F \circ{ }^{k} G\right)^{+}(a) & =(F \circ G)(a) \vee \frac{1-k}{2} \\
& =\left(\bigvee_{(y, z) \in A_{a}}(F(y) \wedge G(z))\right) \vee \frac{1-k}{2} \\
& =\bigvee_{(y, z) \in A_{a}}((F(y) \wedge G(z))) \vee \frac{1-k}{2} \\
& =\bigvee_{(y, z) \in A_{a}}\left(\left(F(y) \vee \frac{1-k}{2}\right) \wedge\left(G(z) \vee \frac{1-k}{2}\right)\right) \\
& =\bigvee_{(y, z) \in A_{a}}\left(F^{+k}(y) \wedge G^{+k}(z)\right) \\
& =\left(F^{+k} \circ G^{+k}\right)(a) .
\end{aligned}
$$

Let $A$ be a non-empty subset of $S$, then the upper part of the characteristic function $\chi_{A}^{k}$ is defined as follows:

$$
\chi_{A}^{+k}: S \longrightarrow[0,1] \mid x \longmapsto \chi_{A}^{+k}(x)= \begin{cases}1, & \text { if } x \in A, \\ \frac{1-k}{2}, & \text { otherwise. }\end{cases}
$$

Lemma 4.3. Let $A$ and $B$ be non-empty subset of $S$. Then the following hold:

(i) $\left(\chi_{A} \wedge^{k} \chi_{B}\right)^{+}=\chi_{A \cap B}^{+k}$,

(ii) $\left(\chi_{A} \vee^{k} \chi_{B}\right)^{+}=\chi_{A \cup B}^{+k}$,

(iii) $\left(\chi_{A} \circ^{k} \chi_{B}\right)^{+}=\chi_{(A B]}^{+k}$.

Proof. The proofs of (i) and (ii) are obvious.

(iii) Let $x \in(A B]$ then $\chi_{(A B]}(x)=1$ and hence $\chi_{(A B]}^{+k}(x)=1 \vee \frac{1-k}{2}=$ 1. Since $x \in(A B]$, we have $x \leq a b$ for some $a \in A$ and $b \in B$. Then 
$(a, b) \in A_{x}$ and $A_{x} \neq \emptyset$. Thus

$$
\begin{aligned}
\left(\chi_{A} \circ{ }^{k} \chi_{B}\right)^{+}(x) & =\left(\chi_{A} \circ \chi_{B}\right)(x) \vee \frac{1-k}{2} \\
& =\left[\bigvee_{(y, z) \in A_{x}}\left(\chi_{A}(y) \wedge \chi_{B}(z)\right)\right] \vee \frac{1-k}{2} \\
& \geq\left(\chi_{A}(a) \wedge \chi_{B}(b)\right) \vee \frac{1-k}{2} .
\end{aligned}
$$

Since $a \in A$ and $b \in B$, we have $\chi_{A}(a)=1$ and $\chi_{B}(b)=1$ and so

$$
\begin{aligned}
\left(\chi_{A} \circ^{k} \chi_{B}\right)^{+}(x) & \geq\left(\chi_{A}(a) \wedge \chi_{B}(b)\right) \vee \frac{1-k}{2} \\
& =(1 \wedge 1) \vee \frac{1-k}{2}=1 .
\end{aligned}
$$

Thus $\left(\chi_{A} \circ^{k} \chi_{B}\right)^{+}(x)=1=\chi_{(A B]}^{+k}(x)$. Let $x \notin(A B]$, then $\chi_{(A B]}(x)=$ 0 and hence, $\chi_{(A B]}^{+k}(x)=0 \vee \frac{1-k}{2}=\frac{1-k}{2}$. Let $(y, z) \in A_{x}$. Then

$$
\begin{aligned}
\left(\chi_{A} \circ{ }^{k} \chi_{B}\right)^{+}(x) & =\left(\chi_{A} \circ \chi_{B}\right)(x) \vee \frac{1-k}{2} \\
& =\left[\bigvee_{(y, z) \in A_{x}}\left(\chi_{A}(y) \wedge \chi_{B}(z)\right)\right] \vee \frac{1-k}{2} .
\end{aligned}
$$

Since $(y, z) \in A_{x}$, then $x \leq y z$. If $y \in A$ and $z \in B$, we have $y z \in A B$ and so $x \in(A B]$. This is a contradiction. If $y \notin A$ and $z \in B$, then

$$
\left[\bigvee_{(y, z) \in A_{x}}\left(\chi_{A}(y) \wedge \chi_{B}(z)\right)\right] \vee \frac{1-k}{2}=\left[\bigvee_{(y, z) \in A_{x}}(0 \wedge 1)\right] \vee \frac{1-k}{2}=\frac{1-k}{2} \text {. }
$$

Hence, $\chi_{(A B]}^{+k}(x)=\frac{1-k}{2}=\left(\chi_{A} \circ^{k} \chi_{B}\right)^{+}(x)$. Similarly, for $y \in A$ and $z \notin B$, we have $\chi_{(A B]}^{+k}(x)=0=\left(\chi_{A} \circ^{k} \chi_{B}\right)^{+}(x)$.

Lemma 4.4. The upper part $\chi_{A}^{+k}$ of the characteristic function $\chi_{A}$ of $A$ is a $\left(\bar{\epsilon}, \bar{\epsilon} \vee \bar{q}_{k}\right)$-fuzzy generalized bi-ideal of $S$ if and only if $A$ is a generalized bi-ideal of $S$.

Proof. Let $A$ be a generalized bi-ideal of $S$. Then by Theorem 2.4 and 3.19, $\chi_{A}^{+k}$ is an $\left(\bar{\epsilon}, \bar{\epsilon} \vee \bar{q}_{k}\right)$-fuzzy generalized bi-ideal of $S$. Conversely, assume that $\chi_{A}^{+k}$ is an $\left(\bar{\epsilon}, \bar{\epsilon} \vee \bar{q}_{k}\right)$-fuzzy generalized bi-ideal of $S$. Let $x, y \in S, x \leq y$. If $y \in A$, then $\chi_{A}^{+k}(y)=1$. Since $\chi_{A}^{+k}$ is an $\left(\bar{\epsilon}, \bar{\epsilon} \vee \bar{q}_{k}\right)$ fuzzy generalized bi-ideal of $S$ and $x \leq y$, we have, $\chi_{A}^{+k}(x) \geq \chi_{A}^{+k}(y)=1$. 
It follows that $\chi_{A}^{+k}(x)=1$ and so $x \in A$. Let $x, z \in A$ and $y \in S$. Then, $\chi_{A}^{+k}(x)=1$ and $\chi_{A}^{+k}(z)=1$. Now,

$$
\chi_{A}^{+k}(x y z) \geq \chi_{A}^{+k}(x) \wedge \chi_{A}^{+k}(z)=1 .
$$

Hence $\chi_{A}^{+k}(x y z)=1$ and so $x y z \in A$. Therefore $A$ is a generalized bi-ideal of $S$.

Lemma 4.5. The upper part $\chi_{A}^{+k}$ of the characteristic function $\chi_{A}$ of $A$ is a $\left(\bar{\epsilon}, \bar{\epsilon} \vee \bar{q}_{k}\right)$-fuzzy left (right)-ideal of $S$ if and only if $A$ is a left (right)-ideal of $S$.

Proof. The proof follows from Lemma 4.4.

In the following proposition, we show that if $F$ is a $\left(\bar{\epsilon}, \bar{\epsilon} \vee \bar{q}_{k}\right)$-fuzzy generalized bi-ideal of $S$, then $F^{+k}$ is a fuzzy generalized bi-ideal of $S$.

Proposition 4.6. If $F$ is a $\left(\bar{\epsilon}, \bar{\epsilon} \vee \bar{q}_{k}\right)$-fuzzy generalized bi-ideal of $S$, then $F^{+k}$ is a fuzzy generalized bi-ideal of $S$.

Proof. Let $x, y \in S, x \leq y$. Since $F$ is an $\left(\bar{\epsilon}, \bar{\epsilon} \vee \bar{q}_{k}\right)$-fuzzy generalized bi-ideal of $S$ and $x \leq y$, then we have $F^{+k}(x)=F(x) \vee \frac{1-k}{2} \geq F(y)$. It follows that $F^{+k}(x)=F(x) \vee \frac{1-k}{2}=\left(F(x) \vee \frac{1-k}{2}\right) \vee \frac{1-k}{2} \geq F(y) \vee \frac{1-k}{2}=$ $F^{+k}(y)$.

For $x, y, z \in S$, we have $F^{+k}(x y z)=F(x y z) \vee \frac{1-k}{2} \geq F(x) \wedge F(z)$. Then

$$
\begin{aligned}
F^{+k}(x y z) & =F(x y z) \vee \frac{1-k}{2}=\left(F(x y z) \vee \frac{1-k}{2}\right) \vee \frac{1-k}{2} \\
& \geq(F(x) \wedge F(z)) \vee \frac{1-k}{2} \\
& =\left(F(x) \vee \frac{1-k}{2}\right) \wedge\left(F(z) \vee \frac{1-k}{2}\right) \\
& =F^{+k}(x) \wedge F^{+k}(z) .
\end{aligned}
$$

Consequently, $F^{+k}$ is a fuzzy generalized bi-ideal of $S$.

In [33], regular and left weakly regular ordered semigroups are characterized by the properties of their fuzzy left (right) and fuzzy generalized bi-ideals. In the following we characterize regular, left weakly regular, left and right simple and completely regular ordered semigroups in terms of $\left(\bar{\epsilon}, \bar{\in} \vee \overline{\mathrm{q}}_{k}\right)$-fuzzy left (right) and $\left(\bar{\epsilon}, \bar{\in} \vee \overline{\mathrm{q}}_{k}\right)$-fuzzy generalized bi-ideals. We first need the following result.

Proposition 4.7. ([23]) An ordered semigroup $S$ is left (right) simple if and only if $(S a]=S((a S]=S)$ for every $a \in S$. 
Proposition 4.8. If $S$ is regular, left and right simple ordered semigroup then for every $\left(\bar{\epsilon}, \bar{\epsilon} \vee \bar{q}_{k}\right)$-fuzzy generalized bi-ideal $F$ of $S$ we have $F^{+k}(a)=F^{+k}(b)$, for every $a, b \in S$.

Proof. Assume that $S$ is regular, left and right simple and $F$ a $(\bar{\epsilon}, \bar{\in} \mathrm{V}$ $\overline{\mathrm{q}}_{k}$ )-fuzzy generalized bi-ideal of $S$. We consider, $E_{S}=\left\{e \in S \mid e \leq e^{2}\right\}$, then $E_{S}$ is obviously non-empty. Let $s, t \in E_{S}$. Since $S$ is left and right simple, by Proposition 4.7, it follows that $S=(S s]$ and $S=(t S]$. Since $t \in S$, we have $t \in(S s]$ and $t \in(s S]$, then $t \leq x s$ and $t \leq s y$ for some $x, y \in S$, and we have

$$
t^{2} \leq(s y)(x s)=s(y x) s .
$$

Since $F$ is a $\left(\bar{\epsilon}, \bar{\epsilon} \vee \overline{\mathrm{q}}_{k}\right)$-fuzzy generalized bi-ideal of $S$, we have

$$
\begin{aligned}
F\left(t^{2}\right) \vee \frac{1-k}{2} & \geq F(s(y x) s) \vee \frac{1-k}{2} \\
& \geq(F(s) \wedge F(s)) \\
& =F(s) .
\end{aligned}
$$

Thus $F^{+k}\left(t^{2}\right)=F\left(t^{2}\right) \vee \frac{1-k}{2}=\left(F\left(t^{2}\right) \vee \frac{1-k}{2}\right) \vee \frac{1-k}{2} \geq F(s) \vee \frac{1-k}{2}=$ $F^{+k}(s)$ and we have

$$
\stackrel{+k}{F}\left(t^{2}\right) \geq \stackrel{+k}{F}(s) .
$$

Since $t \in E_{S}$, we have $t \leq t^{2}$ and so $F(t) \vee \frac{1-k}{2} \geq F\left(t^{2}\right)$. It follows that

$$
\begin{aligned}
F^{+k}(t) & =\left(F(t) \vee \frac{1-k}{2}\right) \vee \frac{1-k}{2} \\
& \geq F\left(t^{2}\right) \vee \frac{1-k}{2},
\end{aligned}
$$

and so $F^{+k}(t) \geq F^{+k}\left(t^{2}\right)$. Thus, by (I), we have $F^{+k}(t) \geq F^{+k}(s)$. On the other hand, since $t \in S$, by Proposition 4.7, we have $(S t]=S=(t S]$. Since $s \in S$, we have $s \in(S t]$ and $s \in(t S]$, then $s \leq a t$ and $s \leq t b$ for some $a, b \in S$. Thus, by the same arguments as above, we get $F^{+k}(s) \geq F^{+k}(t)$. It follows that $F^{+k}(t)=F^{+k}(s)$ and hence $F^{+k}$ is constant on $E_{S}$.

Now, let $a \in S$. Then there exists $x \in S$ such that $a \leq a x a$. It follows that

$$
\begin{aligned}
a x & \leq(a x a) x \\
& =(a x)(a x) \\
& =(a x)^{2},
\end{aligned}
$$


and

$$
\begin{aligned}
x a & \leq x(a x a) \\
& =x a)(x a) \\
& =(x a)^{2} .
\end{aligned}
$$

Thus, $a x, x a \in E_{S}$. By previous arguments, we get, $F^{+k}(a x)=F^{+k}(b)=$ $F^{+k}(x a)$. Since $(a x) a(x a)=(a x a) x a \geq a x a \geq a$, we have

$$
\begin{aligned}
F^{+k}(a) & =F(a) \vee \frac{1-k}{2} \geq F((a x) a(x a)) \vee \frac{1-k}{2} \\
& \geq(F(a x) \wedge F(x a)) .
\end{aligned}
$$

Thus

$$
\begin{aligned}
F^{+k}(a) & =F(a) \vee \frac{1-k}{2}=\left(F(a) \vee \frac{1-k}{2}\right) \vee \frac{1-k}{2} \\
& \geq(F(a x) \wedge F(x a)) \vee \frac{1-k}{2} \\
& =\left(F(a x) \vee \frac{1-k}{2}\right) \wedge\left(F(x a) \vee \frac{1-k}{2}\right),
\end{aligned}
$$

and thus $F^{+k}(a) \geq F^{+k}(a x) \wedge F^{+k}(x a)=F^{+k}(b)$. Since $b \in(S a]$ and $b \in(a S]$, we have $b \leq p a$ and $b \leq a q$ for some $p, q \in S$. Then $b^{2} \leq$ $(a q)(p a)=a(q p) a$ and thus

$$
\begin{aligned}
F\left(b^{2}\right) \vee \frac{1-k}{2} & \geq F(a(q p) a) \\
& \geq(F(a) \wedge F(a)) \\
& =F(a) .
\end{aligned}
$$

Hence, $F^{+k}\left(b^{2}\right)=F\left(b^{2}\right) \vee \frac{1-k}{2}=\left(F\left(b^{2}\right) \vee \frac{1-k}{2}\right) \vee \frac{1-k}{2} \geq F(a) \vee \frac{1-k}{2}$ and we have, $F^{+k}\left(b^{2}\right) \geq F^{+k}(a)$. Since $b \in E_{S}, b^{2} \geq b$, then $F(b) \vee \frac{1-k}{2} \geq$ $F\left(b^{2}\right)$ and hence $\stackrel{+k}{F}(b)=F(b) \vee \frac{1-k}{2}=\left(F(b) \vee \frac{1-k}{2}\right) \vee \frac{1-k}{2} \geq F\left(b^{2}\right) \vee$ $\frac{1-k}{2}$, it follows that $F^{+k}(b) \geq F^{+k}\left(b^{2}\right)$ and so $F^{+k}(b) \geq F^{+k}(a)$. Thus, $F^{+k}(b)=F^{+k}(a)$ and so, $F^{+k}$ is a constant function on $S$.

Proposition 4.9. ([23]) An ordered semigroup $S$ is completely regular if and only if for every $A \subseteq S$, we have $A \subseteq\left(A^{2} S A^{2}\right]$.

Proposition 4.10. Let $S$ be a completely regular ordered semigroup. Then for every $\left(\bar{\epsilon}, \bar{\epsilon} \vee \bar{q}_{k}\right)$-fuzzy generalized bi-ideal $F$ of $S$, we have

$$
F^{+k}(a)=F^{+k}\left(a^{2}\right) \text { for every } a \in S .
$$


Proof. Let $a \in S$. Since $S$ is completely regular, by Proposition 4.9, $a \in\left(a^{2} S a^{2}\right]$. Then there exists $x \in S$ such that $a \leq a^{2} x a^{2}$. Since $F$ is a $\left(\bar{\epsilon}, \bar{\epsilon} \vee \overline{\mathrm{q}}_{k}\right)$-fuzzy generalized bi-ideal of $S$, we have

$$
\begin{aligned}
F(a) \vee \frac{1-k}{2} & \geq F\left(a^{2} x a^{2}\right) \vee \frac{1-k}{2} \\
& \geq\left(F\left(a^{2}\right) \wedge F\left(a^{2}\right)\right) \\
& =F\left(a^{2}\right) \\
& \geq(F(a) \wedge F(a)) \\
& =F(a) .
\end{aligned}
$$

Thus, $F^{+k}(a)=F(a) \vee \frac{1-k}{2}=\left(F(a) \vee \frac{1-k}{2}\right) \vee \frac{1-k}{2} \geq F\left(a^{2}\right) \vee \frac{1-k}{2} \geq$ $F(a) \vee \frac{1-k}{2}$, and it follows that $F^{+k}(a) \geq F^{+k}\left(a^{2}\right) \geq F^{+k}(a)$. Thus $F^{+k}(a)=F^{+k}\left(a^{2}\right)$ for every $a \in S$.

Theorem 4.11. If every $\left(\bar{\epsilon}, \bar{\epsilon} \vee \bar{q}_{k}\right)$-fuzzy generalized bi-ideal $F$ of $S$ satisfies the condition, $F^{+k}(t)=F^{+k}\left(t^{2}\right)$ for every $t \in S$. Then $S$ is completely regular.

Proof. Let $t \in S$. We consider the generalized bi-ideal $B\left(t^{2}\right)=\left(t^{2} \cup\right.$ $\left.t^{2} S t^{2}\right]$ of $S$, generated by $t^{2}(t \in S)$. Then by Lemma 4.4 ,

$$
\stackrel{+}{\chi}_{B\left(t^{2}\right)}^{k}(t)= \begin{cases}1, & \text { if } t \in B\left(t^{2}\right), \\ \frac{1-k}{2}, & \text { otherwise, }\end{cases}
$$

is a $\left(\bar{\epsilon}, \bar{\epsilon} \vee \bar{q}_{k}\right)$-fuzzy generalized bi-ideal of $S$. By hypothesis, we have

$$
\bar{\chi}_{B\left(t^{2}\right)}^{k}\left(t^{2}\right)=\bar{\chi}_{B\left(t^{2}\right)}^{k}(t) \text {. }
$$

Since $t^{2} \in B\left(t^{2}\right)$, we have $\chi_{B\left(t^{2}\right)}^{+k}\left(t^{2}\right)=1$ and hence, $\chi_{B\left(t^{2}\right)}^{+k}(t)=1$, thus $t \in B\left(t^{2}\right)$ and hence, $t \leq t^{2}$ or $t \leq t^{2} x t^{2}$. If $t \leq t^{2}$, then $t \leq t^{2}=t t \leq$ $t^{2} t^{2}=t t t^{2} \leq t^{2} t t^{2} \in t^{2} S t^{2}$ and $t \in\left(t^{2} S t^{2}\right.$ ]. If $t \leq t^{2} x t^{2}$, then $t \in t^{2} S t^{2}$ and $t \in\left(t^{2} S t^{2}\right]$. Thus, $S$ is completely regular.

Proposition 4.12. ([22]) Let $S$ be an ordered semigroup, then the following conditions are equivalent:

(i) $S$ is regular,

(ii) $B \cap L \subseteq(B L]$ for every generalized bi-ideal $B$ and left ideal $L$ of $S$,

(iii) $B(a) \cap L(a) \subseteq(B(a) L(a)]$ for every $a \in S$.

Theorem 4.13. An ordered semigroup $S$ is regular if and only if for every $\left(\bar{\epsilon}, \bar{\in} \vee \bar{q}_{k}\right)$-fuzzy generalized bi-ideal $F$ and $\left(\bar{\epsilon}, \bar{\epsilon} \vee \bar{q}_{k}\right)$-fuzzy left 
ideal $G$ of $S$, we have

$$
\left(F \wedge^{k} G\right)^{+} \preceq\left(F \circ^{k} G\right)^{+} .
$$

Proof. Suppose that $F$ is a $\left(\bar{\epsilon}, \bar{\epsilon} \vee \overline{\mathrm{q}}_{k}\right)$-fuzzy generalized bi-ideal and $G$ a $\left(\bar{\epsilon}, \bar{\in} \vee \bar{q}_{k}\right)$-fuzzy left ideal of a regular ordered semigroup $S$. Let $a \in$ $S$. Since $S$ is regular, there exists $x \in S$ such that $a \leq a x a \leq(a x a)(x a)$. Then $(a x a, x a) \in A_{a}$ and $A_{a} \neq \emptyset$. Thus,

$$
\begin{aligned}
\left(F \circ \circ^{k} G\right)^{+}(a) & =(F \circ G)(a) \vee \frac{1-k}{2} \\
& =\left[\bigvee_{(y, z) \in A_{a}}(F(y) \wedge G(z))\right] \vee \frac{1-k}{2} \\
& \geq(F(a x a) \wedge G(x a)) \vee \frac{1-k}{2} \\
& =\left(F(a x a) \vee \frac{1-k}{2}\right) \wedge\left(G(x a) \vee \frac{1-k}{2}\right) \\
& =F^{+k}(a x a) \wedge G^{+k}(x a) .
\end{aligned}
$$

Since $F$ is a $\left(\bar{\epsilon}, \bar{\epsilon} \vee \overline{\mathrm{q}}_{k}\right)$-fuzzy generalized bi-ideal and $G$ an $\left(\bar{\epsilon}, \bar{\epsilon} \vee \overline{\mathrm{q}}_{k}\right)$ fuzzy left ideal of $S$, we have $F^{+k}(a x a) \geq F^{+k}(a) \wedge F^{+k}(a)=F^{+k}(a)$ and $G^{+k}(x a) \geq G^{+k}(a)$. Therefore,

$$
\left[F^{+k}(a x a) \wedge G^{+k}(x a)\right] \geq F^{+k}(a) \wedge G^{+k}(a)=\left(F \wedge^{k} G\right)^{+}(a) .
$$

Thus $\left(F \circ^{k} G\right)^{+}(a) \geq\left(F \wedge^{k} G\right)^{+}(a)$.

Conversely, assume that $\left(F \wedge^{k} G\right)^{+} \preceq\left(F \circ^{k} G\right)^{+}$for every $\left(\bar{\epsilon}, \bar{\epsilon} \vee \overline{\mathrm{q}}_{k}\right)$ fuzzy generalized bi-ideal $F$ and every $\left(\bar{\epsilon}, \bar{\epsilon} \vee \bar{q}_{k}\right)$-fuzzy left ideal $G$ of $S$. To prove that $S$ is regular, by Proposition 4.12 it is enough to prove that,

$B \cap L \subseteq(B L]$ for generalized bi-ideal $B$ and left ideal $L$ of $S$.

Let $x \in B \cap L$. Then $x \in B$ and $x \in L$. Since $B$ is a generalized bi-ideal and $L$ a left ideal of $S$, by Lemma 4.4 and $4.5, \chi_{B}^{+k}$ is a $\left(\bar{\epsilon}, \bar{\epsilon} \vee \bar{q}_{k}\right)$ fuzzy generalized bi-ideal and $\chi_{L}^{+k}$ a $\left(\bar{\epsilon}, \bar{\epsilon} \vee \overline{\mathrm{q}}_{k}\right)$-fuzzy left ideal of $S$. By hypothesis, $\left(\chi_{B} \circ^{k} \chi_{L}\right)^{+}(x) \geq\left(\chi_{B} \wedge^{k} \chi_{L}\right)^{+}(x)=\left(\chi_{B}^{k} \wedge \chi_{L}^{k}\right)(x) \vee \frac{1-k}{2}$. Since $x \in B$ and $x \in L$, we have $\chi_{B}^{+k}(x)=1$ and $\chi_{L}^{+k}(x)=1$. Thus $\left(\chi_{B}^{+k} \wedge \chi_{L}^{+k}\right)(x)=1$. It follows that $\left(\chi_{B} \circ^{k} \chi_{L}\right)^{+}(x)=1$. By using Lemma 4.3 (iii), we have $\left(\chi_{B} \circ^{k} \chi_{L}\right)^{+}=\chi_{(B L]}^{+k}$. Therefore, $\chi_{(B L]}^{+k}(x)=1$ and so $x \in(B L]$. Consequently, $S$ is regular. 
Proposition 4.14. ([22]) Let $S$ be an ordered semigroup, then the following conditions are equivalent:

(i) $S$ is regular,

(ii) $B \cap I=(B I B]$ for every generalized bi-ideal $B$ and ideal $I$ of $S$,

(iii) $B(a) \cap I(a)=(B(a) I(a) B(a)]$ for every $a \in S$.

Theorem 4.15. An ordered semigroup $S$ is regular if and only if for every $\left(\in, \in \vee q_{k}\right)$-fuzzy generalized bi-ideal $F$ and every $\left(\bar{\epsilon}, \bar{\epsilon} \vee \bar{q}_{k}\right)$-fuzzy ideal $G$ of $S$, we have

$$
\left(F \wedge^{k} G\right)^{+} \preceq\left(F \circ^{k} G \circ^{k} F\right)^{+} .
$$

Proof. Suppose that $F$ is a $\left(\bar{\epsilon}, \bar{\epsilon} \vee \overline{\mathrm{q}}_{k}\right)$-fuzzy generalized bi-ideal and $G$ a $\left(\bar{\epsilon}, \bar{\epsilon} \vee \overline{\mathrm{q}}_{k}\right)$-fuzzy ideal of a regular ordered semigroup $S$. Let $a \in S$. Since $S$ is regular, there exits $x \in S$ such that $a \leq a x a \leq(a x a)(x a)=$ $a(x a x a)$. Then $(a, x a x a) \in A_{a}$ and $A_{a} \neq \emptyset$. Thus,

$$
\begin{aligned}
& \left(F \circ^{k} G \circ^{k} F\right)^{+}(a) \\
= & \left(F \circ^{k} G \circ F\right)(a) \vee \frac{1-k}{2} \\
= & {\left[\bigvee_{(y, z) \in A_{a}}\left(F \circ^{k} G\right)(y) \wedge F(z)\right] \vee \frac{1-k}{2} } \\
= & \bigvee_{(y, z) \in A_{a}}\left[\bigvee_{(p, q) \in A_{a}}\left((F(p) \wedge G(q)) \vee \frac{1-k}{2}\right) \wedge F(z)\right] \vee \frac{1-k}{2} \\
= & \bigvee_{(y, z) \in A_{a}} \bigvee_{(p, q) \in A_{a}}((F(p) \wedge G(q)) \wedge F(z)) \vee \frac{1-k}{2} \\
= & \bigvee_{(y, z) \in A_{a}} \bigvee_{(p, q) \in A_{a}}(F(p) \wedge G(q) \wedge F(z)) \vee \frac{1-k}{2} \\
\geq & (F(a) \wedge G(x a x) \wedge F(a)) \vee \frac{1-k}{2} .
\end{aligned}
$$

Since $G$ a $\left(\bar{\epsilon}, \bar{\epsilon} \vee \overline{\mathrm{q}}_{k}\right)$-fuzzy ideal of $S$, we have $G(x a x) \vee \frac{1-k}{2} \geq$ $G(a x) \wedge \frac{1-k}{2} \geq G(a)$. Therefore

$$
\begin{aligned}
& {\left[F(a) \wedge G(\text { xax }) \wedge F(a) \wedge \frac{1-k}{2}\right] } \\
= & {\left[F(a) \wedge\left(G(x a x) \vee \frac{1-k}{2}\right) \wedge F(a) \vee \frac{1-k}{2}\right] } \\
\geq & {\left[F(a) \wedge G(a) \wedge F(a) \vee \frac{1-k}{2}\right] }
\end{aligned}
$$




$$
\begin{aligned}
& \geq\left(F(a) \vee \frac{1-k}{2}\right) \wedge\left(G(a) \vee \frac{1-k}{2}\right) \\
& =F^{+}(a) \wedge^{k} G^{+}(a) .
\end{aligned}
$$

Thus $\left(F \circ^{k} G \circ^{k} F\right)^{+}(a) \geq\left(F \wedge^{k} G\right)^{+}(a)$.

Conversely, assume that $\left(F \wedge^{k} G\right)^{+} \preceq\left(F \circ^{k} G \circ^{k} F\right)^{+}$for every $(\bar{\epsilon}$, $\left.\bar{\epsilon} \vee \overline{\mathrm{q}}_{k}\right)$-fuzzy generalized bi-ideal $F$ and every $\left(\bar{\epsilon}, \bar{\epsilon} \vee \overline{\mathrm{q}}_{k}\right)$-fuzzy ideal $F$ of $S$. To prove that $S$ is regular, by Proposition 4.14, it is enough to prove that $B \cap I \subseteq(B I B]$ for generalized bi-ideal $B$ and ideal $I$ of $S$.

Let $x \in B \cap I$. Then $x \in B$ and $x \in I$. Since $B$ is a generalized biideal and $I$ an ideal of $S$, by Lemma 4.4 and $4.5, \chi_{B}^{+k}$ is an $\left(\bar{\epsilon}, \bar{\epsilon} \vee \overline{\mathrm{q}}_{k}\right)$ fuzzy generalized bi-ideal and $\chi_{I}^{+k}$ an $\left(\bar{\epsilon}, \bar{\epsilon} \vee \overline{\mathrm{q}}_{k}\right)$-fuzzy ideal of $S$. By hypothesis, $\left(\chi_{B} \circ^{k} \chi_{I}{ }^{k} \chi_{B}\right)^{+}(x) \geq\left(\chi_{B} \wedge{ }^{k} \chi_{I}\right)^{+}(x)=\left(\chi_{B} \wedge \chi_{I}\right)(x) \vee \frac{1-k}{2}$. Since $x \in B$ and $x \in I$, we have $\chi_{B}^{+k}(x)=1$ and $\chi_{I}^{+k}(x)=1$. Thus $\left(\chi_{B} \wedge \chi_{I}\right)(x) \vee \frac{1-k}{2}=1$. It follows that $\left(\chi_{B} 0^{\circ} \chi_{I}{ }^{\circ}{ }^{k} \chi_{B}\right)^{+}(x)=1$. By using Lemma 4.3 (iii), we have $\left(\chi_{B} \circ^{k} \chi_{I} \circ^{k} \chi_{B}\right)^{+}(x)=\chi_{(B I B]}^{+k}(x)$. Therefore, $\chi_{(B I B]}^{+k}(x)=1$ and so $x \in(B I B]$. Consequently, $S$ is regular.

Proposition 4.16. ([22]) Let $S$ be an ordered semigroup, then the following conditions are equivalent:

(i) $S$ is regular,

(ii) $R \cap B \cap L \subseteq(R B L]$ for every right ideal $R$, generalized bi-ideal $B$ and left ideal $L$ of $S$,

(iii) $R(a) \cap B(a) \cap L(a) \subseteq(R(a) B(a) L(a)]$ for every $a \in S$.

Theorem 4.17. An ordered semigroup $S$ is regular if and only if for every $\left(\bar{\epsilon}, \bar{\epsilon} \vee \bar{q}_{k}\right)$-fuzzy generalized bi-ideal $F$, every $\left(\bar{\epsilon}, \bar{\epsilon} \vee \bar{q}_{k}\right)$-fuzzy right ideal $G$ and every $\left(\bar{\epsilon}, \bar{\epsilon} \vee \bar{q}_{k}\right)$-fuzzy left ideal $H$ of $S$, we have,

$$
(F \wedge G \wedge H)^{+} \preceq(G \circ G \circ H)^{+} .
$$

Proof. Let $S$ be a regular ordered semigroup, $F$ a $\left(\bar{\epsilon}, \bar{\epsilon} \vee \overline{\mathrm{q}}_{k}\right)$-fuzzy generalized bi-ideal, $G$ a $\left(\bar{\epsilon}, \bar{\epsilon} \vee \overline{\mathrm{q}}_{k}\right)$-fuzzy right ideal and $H$ a $\left(\bar{\epsilon}, \bar{\epsilon} \vee \overline{\mathrm{q}}_{k}\right)$ fuzzy left ideal of $S$. Let $a \in S$. Since $S$ is regular, there exists $x \in S$ such that $a \leq a x a=a x a \leq(a x a)(x a) \leq(a x a) x(a x a)=(a x)(a x a)(x a)$. Then $((a x)(a x a), x a) \in A_{a}$ and $A_{a} \neq \emptyset$. Thus,

$$
\begin{aligned}
& \left(F \circ^{k} G \circ^{k} H\right)^{+}(a) \\
= & \left(F \circ^{k} G \circ H\right)(a) \vee \frac{1-k}{2}
\end{aligned}
$$




$$
\begin{aligned}
& =\left[\bigvee_{(y, z) \in A_{a}}\left(F \circ^{k} G\right)(y) \wedge H(z)\right] \vee \frac{1-k}{2} \\
& =\bigvee_{(y, z) \in A_{a}}\left[\bigvee_{(p, q) \in A_{a}}\left((F(p) \wedge G(q)) \vee \frac{1-k}{2}\right) \wedge H(z)\right] \vee \frac{1-k}{2} \\
& =\bigvee_{(y, z) \in A_{a}} \bigvee_{(p, q) \in A_{a}}((F(p) \wedge G(q)) \wedge H(z)) \vee \frac{1-k}{2} \\
& =\bigvee_{(y, z) \in A_{a}} \bigvee_{(p, q) \in A_{a}}(F(p) \wedge G(q) \wedge H(z)) \vee \frac{1-k}{2} \\
& \geq(F(a x) \wedge G(a x a) \wedge H(x a)) \vee \frac{1-k}{2} .
\end{aligned}
$$

Since $F$ is a $\left(\bar{\epsilon}, \bar{\epsilon} \vee \overline{\mathrm{q}}_{k}\right)$-fuzzy right ideal $G$, a $\left(\bar{\epsilon}, \bar{\epsilon} \vee \overline{\mathrm{q}}_{k}\right)$-fuzzy generalized ideal and $H$ a $\left(\bar{\epsilon}, \bar{\epsilon} \vee \overline{\mathrm{q}}_{k}\right)$-fuzzy left ideal of $S$, we have $F(a x) \vee$ $\frac{1-k}{2} \geq F(a) \vee \frac{1-k}{2}, G(a x a) \vee \frac{1-k}{2} \geq G(a) \wedge G(a) \vee \frac{1-k}{2}$ and $H(x a) \vee \frac{1-k}{2} \geq$ $H(a) \vee \frac{1-k}{2}$. Therefore,

$$
\begin{aligned}
& {[F(a x) \wedge G(a x a) \wedge H(x a)] \vee \frac{1-k}{2} } \\
= & {\left[\left(F(a x) \vee \frac{1-k}{2}\right) \wedge\left(G(a x a) \vee \frac{1-k}{2}\right) \wedge\left(H(x a) \vee \frac{1-k}{2}\right)\right] } \\
\geq & {\left[\left(F(a) \vee \frac{1-k}{2}\right) \wedge\left(G(a) \vee \frac{1-k}{2}\right) \wedge\left(H(a) \vee \frac{1-k}{2}\right)\right] } \\
= & F^{+}(a) \wedge^{k} G^{+}(a) \wedge^{k} H^{+}(a) .
\end{aligned}
$$

Thus $\left(F \circ^{k} G \circ^{k} H\right)^{+}(a) \geq\left(F \wedge^{k} G \wedge^{k} H\right)^{+}(a)$.

Conversely, assume that $\left(\lambda \wedge^{k} \mu \wedge^{k} \rho\right)^{+} \preceq\left(\lambda \circ^{k} \mu \circ^{k} \rho\right)^{+}$for every $\left(\bar{\epsilon}, \bar{\epsilon} \vee \overline{\mathrm{q}}_{k}\right)$-fuzzy right ideal $F$, every $\left(\bar{\epsilon}, \bar{\epsilon} \vee \overline{\mathrm{q}}_{k}\right)$-fuzzy generalized ideal $G$ and every $\left(\bar{\epsilon}, \bar{\in} \vee \overline{\mathrm{q}}_{k}\right)$-fuzzy left ideal $H$ of $S$. To prove that $S$ is regular, by Proposition 4.16, it is enough to prove that $R \cap B \cap L \subseteq(R B L]$ for right ideal $R$, generalized bi-ideal $B$ and left ideal $L$ of $S$. Let $x \in R \cap B \cap L$. Then $x \in R, x \in B$ and $x \in L$. Since $R$ is a right ideal, $B$ a generalized bi-ideal and $L$ a left ideal of $S$, by Lemma 4.4 and $4.5, \chi_{R}^{+k}$ is an $\left(\bar{\epsilon}, \bar{\epsilon} \vee \overline{\mathrm{q}}_{k}\right)$-fuzzy right ideal, $\chi_{B}^{+k}$ an $\left(\bar{\epsilon}, \bar{\epsilon} \vee \overline{\mathrm{q}}_{k}\right)$-fuzzy generalized biideal and $\chi_{L}^{+k}$ an $\left(\bar{\epsilon}, \bar{\epsilon} \vee \overline{\mathrm{q}}_{k}\right)$-fuzzy left ideal of $S$. By hypothesis, $\left(\chi_{R} \circ^{k}\right.$ $\left.\chi_{B} \circ^{k} \chi_{L}\right)^{+}(x) \geq\left(\chi_{R} \wedge{ }^{k} \chi_{B} \wedge^{k} \chi_{L}\right)^{+}(x)=\left(\chi_{R} \wedge \chi_{B} \wedge \chi_{L}\right)(x) \vee \frac{1-k}{2}$. Since $x \in R, x \in B$ and $x \in L$, we have $\chi_{R}^{+k}=1, \chi_{B}^{+k}=1$ and $\chi_{L}^{+k}=1$. Thus $\left(\chi_{R} \wedge^{k} \chi_{B} \wedge^{k} \chi_{L}\right)^{+}(x)=\left(\chi_{R} \wedge \chi_{B} \wedge \chi_{L}\right)(x) \vee \frac{1-k}{2}=1$. Follows that $\left(\chi_{R} \circ^{k} \chi_{B} \circ^{k} \chi_{L}\right)^{+}(x)=1$. By using Lemma 4.4 (iii), we 
have $\left(\chi_{R} \circ^{k} \chi_{B} \circ^{k} \chi_{L}\right)^{+}=\chi_{(R B L]}^{+k}$. Therefore, $\chi_{(R B L]}^{+k}(x)=1$ and so $x \in(R B L]$. Consequently, $S$ is regular.

Proposition 4.18. ([22]) Let $(S, \cdot, \leq)$ be an ordered semigroup, then the following conditions are equivalent:

(1) $S$ is left weakly regular,

(2) $I \cap L \subseteq(I L]$ for every ideal $I$ and left ideal $L$ of $S$,

(3) $I(a) \cap L(a) \subseteq(I(a) L(a)]$ for every $a \in S$.

Theorem 4.19. An ordered semigroup $(S, \cdot, \leq)$ is left weakly regular if and only if for every $\left(\bar{\epsilon}, \bar{\epsilon} \vee \bar{q}_{k}\right)$-fuzzy ideal $F$ and every $\left(\bar{\epsilon}, \bar{\epsilon} \vee \bar{q}_{k}\right)$ fuzzy left ideal $G$ of $S$,

$$
\left(F \wedge^{k} G\right)^{+} \preceq\left(F \circ^{k} G\right)^{+} .
$$

Proof. Suppose that $F$ is a $\left(\bar{\epsilon}, \bar{\epsilon} \vee \bar{q}_{k}\right)$-fuzzy ideal and $G$ a $\left(\bar{\epsilon}, \bar{\in} \vee \overline{\mathrm{q}}_{k}\right)$ fuzzy left ideal of a left weakly regular ordered semigroup $S$. Let $a \in S$. Since $S$ is left weakly regular, there exists $x, y \in S$ such that $a \leq x a y a=$ $(x a)(y a)$. Then $(x a, y a) \in A_{a}$ and $A_{a} \neq \emptyset$. Thus,

$$
\begin{aligned}
\left(F \circ{ }^{k} G\right)^{+}(a) & =(F \circ G)(a) \vee \frac{1-k}{2} \\
& =\left[\bigvee_{(y, z) \in A_{a}}(F(y) \wedge G(z))\right] \vee \frac{1-k}{2} \\
& \geq(F(x a) \wedge G(y a)) \vee \frac{1-k}{2} .
\end{aligned}
$$

Since $F$ is an $\left(\bar{\epsilon}, \bar{\epsilon} \vee \overline{\mathrm{q}}_{k}\right)$-fuzzy ideal and $G$ an $\left(\bar{\epsilon}, \bar{\epsilon} \vee \overline{\mathrm{q}}_{k}\right)$-fuzzy left ideal of $S$, we have $F(x a) \vee \frac{1-k}{2} \geq F(a) \vee \frac{1-k}{2}$ and $G(y a) \vee \frac{1-k}{2} \geq$ $G(a) \vee \frac{1-k}{2}$. Therefore,

$$
\begin{aligned}
(F(x a) \wedge G(y a)) \vee \frac{1-k}{2} & =\left[\left(F(x a) \vee \frac{1-k}{2}\right) \wedge\left(G(y a) \vee \frac{1-k}{2}\right)\right] \\
& \geq\left(F(a) \vee \frac{1-k}{2}\right) \wedge\left(G(a) \vee \frac{1-k}{2}\right) \\
& =F^{+}(a) \wedge{ }^{k} G^{+}(a) .
\end{aligned}
$$

Thus $\left(F \circ^{k} G\right)^{+}(a) \geq\left(F \wedge^{k} G\right)^{+}(a)$.

Conversely, assume that $\left(\lambda \wedge^{k} \mu\right)^{-} \preceq\left(\lambda \circ^{k} \mu\right)^{-}$for every $\left(\bar{\epsilon}, \bar{\epsilon} \vee \overline{\mathrm{q}}_{k}\right)$ fuzzy ideal $\lambda$ and every $\left(\bar{\epsilon}, \bar{\epsilon} \vee \bar{q}_{k}\right)$-fuzzy left ideal $\mu$ of $S$. To prove that $S$ is left weakly regular, by Proposition 4.18, it is enough to prove that

$I \cap L \subseteq(I L]$ for ideal $I$ and left ideal $L$ of $S$. 
Let $x \in I \cap L$. Then $x \in I$ and $x \in L$. Since $I$ is an ideal and $L$ a left ideal of $S$, by Lemma 4.4 and $4.5, \chi_{I}^{+k}$ is an $\left(\bar{\epsilon}, \bar{\in} \vee \bar{q}_{k}\right)$-fuzzy ideal and $\bar{\chi}_{L}^{k}$ an $\left(\bar{\epsilon}, \bar{\epsilon} \vee \overline{\mathrm{q}}_{k}\right)$-fuzzy left ideal of $S$. By hypothesis, $\left(\chi_{I} \circ^{k} \chi_{L}\right)^{+}(x) \geq$ $\left(\chi_{I} \wedge^{k} \chi_{L}\right)^{+}(x)=\left(\chi_{I}^{k} \wedge \chi_{L}^{k}\right)(x) \vee \frac{1-k}{2}$. Since $x \in I$ and $x \in L$, we have $\chi_{I}^{+k}=1$ and $\chi_{L}^{+k}=1$. Thus $\left(\chi_{I}^{+k} \wedge \chi_{L}^{+k}\right)(x) \vee \frac{1-k}{2}=1$. It follows that $\left(\chi_{I} \circ^{k} \chi_{L}\right)^{+}(x)=1$. By using Lemma 4.3 (iii), we have $\left(\chi_{I} \circ^{k} \chi_{L}\right)^{+}=$ $\chi_{(I L]}^{+k}(x)$. Therefore, $\chi_{(I L]}^{+k}(x)=1$ and so $x \in(I L]$. Consequently, $S$ is left weakly regular.

Proposition 4.20. ([22]) Let $S$ be an ordered semigroup, then the following conditions are equivalent:

(i) $S$ is left weakly regular,

(ii) $I \cap B \subseteq(I B]$ for every generalized bi-ideal $B$ and every ideal $I$ of $S$,

(iii) $I(a) \cap B(a) \subseteq(I(a) B(a)]$ for every $a \in S$.

Theorem 4.21. An ordered semigroup $(S, \cdot, \leq)$ is left weakly regular if and only if for every $\left(\bar{\epsilon}, \bar{\epsilon} \vee \bar{q}_{k}\right)$-fuzzy ideal $F$ and every $\left(\bar{\epsilon}, \bar{\epsilon} \vee \bar{q}_{k}\right)$ fuzzy generalized bi-ideal $G$ of $S$,

$$
\left(F \wedge^{k} G\right)^{+} \preceq\left(F \circ^{k} G\right)^{+} .
$$

Proof. Suppose that $F$ is a $\left(\bar{\epsilon}, \bar{\epsilon} \vee \overline{\mathrm{q}}_{k}\right)$-fuzzy ideal and $G$ a $\left(\bar{\epsilon}, \bar{\epsilon} \vee \overline{\mathrm{q}}_{k}\right)$ fuzzy generalized bi-ideal of a left weakly regular ordered semigroup $S$. Let $a \in S$. Since $S$ is left weakly regular, there exists $x, y \in S$ such that $a \leq x a y a \leq x(x a y a) y a=\left(x^{2} a y\right)(a y a)$. Then $\left(x^{2} a y, a y a\right) \in A_{a}$ and $A_{a} \neq \emptyset$. Thus,

$$
\begin{aligned}
\left(F \circ \circ^{k} G\right)^{+}(a) & =(F \circ G)(a) \vee \frac{1-k}{2} \\
& =\left[\bigvee_{(y, z) \in A_{a}}(F(y) \wedge G(z))\right] \vee \frac{1-k}{2} \\
& \geq\left(F\left(x^{2} a y\right) \wedge G(a y a)\right) \vee \frac{1-k}{2} .
\end{aligned}
$$

Since $F$ is a $\left(\bar{\epsilon}, \bar{\epsilon} \vee \overline{\mathrm{q}}_{k}\right)$-fuzzy ideal and $G$ a $\left(\bar{\epsilon}, \bar{\epsilon} \vee \overline{\mathrm{q}}_{k}\right)$-fuzzy generalized bi-ideal of $S$, we have $F\left(x^{2} a y\right) \vee \frac{1-k}{2} \geq F(a y) \vee \frac{1-k}{2} \geq F(a) \vee \frac{1-k}{2}$ and $G($ aya $) \vee \frac{1-k}{2} \geq(G(a) \wedge G(a)) \vee \frac{1-k}{2}=G(a) \vee \frac{1-k}{2}$. Therefore

$$
\begin{aligned}
& F\left(x^{2} a y\right) \wedge G(a y a) \vee \frac{1-k}{2} \\
= & {\left[\left(F\left(x^{2} a y\right) \vee \frac{1-k}{2}\right) \wedge\left(G(\text { aya }) \vee \frac{1-k}{2}\right)\right] }
\end{aligned}
$$




$$
\begin{aligned}
& \geq\left(F(a) \wedge \frac{1-k}{2}\right) \wedge\left(G(a) \wedge \frac{1-k}{2}\right) \\
& =F^{+}(a) \wedge^{k} G^{+}(a)
\end{aligned}
$$

Thus $\left(F \circ^{k} G\right)^{+}(a) \geq\left(F \wedge^{k} G\right)^{+}(a)$.

Conversely, assume that $\left(\lambda \wedge^{k} \mu\right)^{+} \preceq\left(\lambda \circ^{k} \mu\right)^{+}$for every $\left(\bar{\epsilon}, \bar{\epsilon} \vee \bar{q}_{k}\right)$ fuzzy ideal $F$ and every $\left(\bar{\epsilon}, \bar{\epsilon} \vee \overline{\mathrm{q}}_{k}\right)$-fuzzy generalized bi-ideal $G$ of $S$. To prove that $S$ is left weakly regular, by Proposition 4.20, it is enough to prove that

$$
I \cap B \subseteq(I L] \text { for ideal } I \text { and generalized bi-ideal } B \text { of } S \text {. }
$$

Let $x \in I \cap B$. Then $x \in I$ and $x \in B$. Since $I$ is an ideal and $B$ a generalized bi-ideal of $S$, by Lemma 4.4 and $4.5, \chi_{I}^{+k}$ is an $\left(\bar{\epsilon}, \bar{\epsilon} \vee \bar{q}_{k}\right)$ fuzzy ideal and $\chi_{B}^{+k}$ an $\left(\bar{\epsilon}, \bar{\in} \vee \overline{\mathrm{q}}_{k}\right)$-fuzzy generalized bi-ideal of $S$. By hypothesis, $\left(\chi_{I} \circ^{k} \chi_{B}\right)^{+}(x) \geq\left(\chi_{I} \wedge^{k} \chi_{B}\right)^{+}(x)=\left(\chi_{I} \wedge \chi_{B}\right)(x) \vee \frac{1-k}{2}$. Since $x \in I$ and $x \in B$, we have $\chi_{I}^{+k}(x)=1$ and $\chi_{B}^{+k}(x)=1$. Thus $\left(\chi_{I} \wedge \chi_{B}\right)(x) \vee \frac{1-k}{2}=1$. It follows that $\left(\chi_{I} \circ^{k} \chi_{B}\right)^{+}(x)=1$. By using Lemma 4.3 (iii), we have $\left(\chi_{I} \circ^{k} \chi_{B}\right)^{+}=\chi_{(I B]}^{+k}$. Therefore, $\chi_{(I B]}^{+k}(x)=1$ and so $x \in(I B]$. Consequently, $S$ is left weakly regular.

\section{References}

[1] L. A. Zadeh, Fuzzy sets, Information and control, 8 (1965), 338-3353.

[2] A. Rosenfeld, Fuzzy groups, J. Math. Anal. Appl., 35 (1971), 512-517.

[3] S. K. Bhakat and P. Das, $(\in, \in \vee q)$-fuzzy subgroups, Fuzzy Sets and Systems 80 (1996), 359-368.

[4] P. M. Pu and Y. M. Liu, Fuzzy topology I, neighborhood structure of a fuzzy point and Moore-Smith convergence, J. Math. Anal. Appl., 76 (1980), 571-599.

[5] B. Davvaz and A. Khan, Characterizations of regular ordered semigroups in terms of $(\alpha, \beta)$-fuzzy generalized bi-ideals, Inform. Sci., 181 (2011), 1759-1770.

[6] Y. B. Jun and S. Z. Song, Generalized fuzzy interior ideals in semigroups, Inform. Sci. 176 (2006), 3079-3093.

[7] O. Kazanci and S. Yamak, Generalized fuzzy bi-ideals of semigroup, Soft Computing, 12 (2008), 1119-1124.

[8] M. Shabir, Y. B. Jun and Y. Nawaz, Semigroups characterized by $(\alpha, \beta)$-fuzzy ideals, Computer and Mathematics with Applications 59 (2010), 161-175.

[9] M. Shabir, Y. B. Jun and Y. Nawaz, Characterization of regular semigroups by $\left(\in, \in \vee q_{k}\right)$-fuzzy ideals, Computer and Mathematics with Applications 60 (2010), 1473-1493.

[10] M. Shabir, Y. Nawaz and M. Ali, Characterizations of Semigroups by $\left(\bar{\epsilon}, \bar{\epsilon} \vee \overline{\mathrm{q}}_{k}\right)$ fuzzy Ideals, World Applied Sciences, 14(12) (2011), 1866-1878. 
[11] B. Davvaz, O. Kazanci and S. Yamak, Generalized fuzzy n-ary subpolygroups endowed with interval valued membership functions, Journal Intelligent and Fuzzy Systems, 20(4-5) (2009), 159-168.

[12] B. Davvaz and Z. Mozafar, $(\in, \in \vee q)$-fuzzy Lie subalgebra and ideals, International Journal of Fuzzy Systems, 11(2) (2009), 123-129.

[13] B. Davvaz and P. Corsini, On $(\alpha, \beta)$-fuzzy $H_{v}$-ideals of $H_{v}$-rings, Iranian Journal of Fuzzy System 5 (2008), 35-47.

[14] B. Davvaz, J. Zhan, K.P. Shum, Generalized fuzzy polygroups endowed with interval valued membership functions, Journal of Intelligent and Fuzzy Systems, 19(3) (2008), 181-188.

[15] B. Davvaz, Fuzzy R-subgroups with thresholds of near-rings and implication operators, Soft Computing, 12 (2008), 875-879.

[16] O. Kazanci and B. Davvaz, Fuzzy n-ary polygroups related to fuzzy points, Computers \& Mathematics with Applications, 58(7) (2009), 1466-1474.

[17] X. Ma, J. Zhan and Y. B. Jun, Interval valued $(\in, \in \vee q)$-fuzzy ideals of pseudoMV algebras, International Journal of Fuzzy Systems, 10(2) (2008), 84-91.

[18] J. N. Mordeson, D. S. Malik and N. Kuroki, Fuzzy Semigroups, Studies in Fuzziness and Soft Computing, Vol. 131, Springer-Verlag Berlin (2003).

[19] Y. Yin and J. Zhan, New types of fuzzy filters of BL-algebras, Computer Mathematics with Applications, 60 (2010), 2115-2125.

[20] X. Yuan, C. Zhang and Y. Ren, Generalized fuzzy groups and many-valued implications, Fuzzy Sets and Systems, 138 (2003), 205-211.

[21] J. Zhan, B. Davvaz, K. P. Shum, A new view of fuzzy hyperquasigroups, Journal of Intelligent and Fuzzy Systems, 20 (2009), 147-157.

[22] M. Shabir, A. Khan, Characterizations of ordered semigroups by the properties of their fuzzy generalized bi-ideals, New Mathematics and Natural Computation, 4(2) (2008), 237-250.

[23] N. Kehayopulu and M. Tsingelis, Fuzzy bi-ideals in ordered semigroups, Information Sciences, 171 (2005), 13-28.

[24] N. Kuroki, On fuzzy ideals and fuzzy bi-ideals in semigroups, Fuzzy Sets and Systems, 5 (1981), 203-215.

[25] N. Kuroki, Fuzzy congruences on $T^{*}$-pure semigroups, Information Sciences, 84 (1995), 239-246.

[26] N. Kuroki, Fuzzy semiprime quasi-ideals in semigroups, Information Sciences, 75 (1993), 201-211.

[27] N. Kuroki, On fuzzy semigroups, Information Sciences, 53 (1991), 203-236.

[28] A. Khan, Y. B. Jun, Z. Abbasi, Characterizations of ordered semigroups in terms of $(\in, \in \vee q)$-fuzzy interior ideals, Neural Comp., Appli., 21(3) (2012), 433-440.

Hidayat Ullah Khan

Department of Mathematical Sciences, Faculty of Science, Universiti Teknologi Malaysia, 81310 UTM Johor Bahru, Johor, Malaysia. 
E-mail: hidayatullak@yahoo.com

Nor Haniza Sarmin

Department of Mathematical Sciences, Faculty of Science, Universiti Teknologi Malaysia,

81310 UTM Johor Bahru, Johor, Malaysia.

E-mail: nhs@utm.my

Asghar Khan

Department of Mathematics, Abdul Wali Khan University Mardan, Mardan, Khyber Pakhtunkhwa, Pakistan.

E-mail: azhar4set@yahoo.com 